\title{
An $x$-ray microscopic study of the postnatal development of the vasa vasorum in the pulmonary trunk and arteries
}

\author{
J O H N A. CLARK E ${ }^{1}$ \\ From the Department of Anatomy, University of Glasgow, Glasgow, W.2
}

Little reference could be found in the literature to the development of the vasa vasorum in the pulmonary arterial wall of neonates, infants, or children.

The main paper on the vasa vasorum of the arterial wall during this period of life is by Schlichter (1948), who $x$-rayed (technical details not given) $10 \mu$ thick sections of human, dog, rabbit, and chicken aorta after the injection of a radioopaque medium, and concluded that, although the dog aorta was the most vascular, the human aorta received the richest distribution of vasa vasorum during the neonatal period. Winternitz, Thomas, and LeCompte (1938) state, without further amplification or illustration, that the vasa vasorum of the 'pulmonary artery' communicate with the vasa vasorum of the ductus arteriosus.

The present work is part of a series on the vasa vasorum of the human arterial wall using the Coslett Nixon $x$-ray projection microscope. In a previous communication the pattern of the vasa vasorum in adult pulmonary arteries was described (Clarke, 1964), and $x$-ray microscopy has been discussed as a method for investigating small arteriole and capillary beds (Clarke, 1965a), the results being compared with the routine injection and clearing techniques of previous investigators (Pickworth, 1934 ; Winternitz et al., 1938 ; O’Neill, 1947 ; Williams, 1948 ; Scharrer, 1950 ; Woerner, 1959).

\section{MATERIAL AND METHODS}

Normal pulmonary trunks and arteries were obtained within 12 hours of death from 20 neonates and from 20 children in the first five years of life. They were examined in equally divided groups of five at weekly and yearly intervals respectively.

Fifteen normal pulmonary trunks and arteries were obtained as before from children between 5 and 15

\footnotetext{
1 Present address: Department of Anatomy, St. Bartholomew's Hospital Medical College, London, E.C.1.
}

years of age and were examined in equally divided groups of five at three-yearly intervals. In no case was there a history or evidence of pulmonary or cardio- -5 vascular disease.

In each case the pulmonary trunk and arteries, with $\subseteq$ the heart attached, were removed in a single block of tissue and the bronchial arteries were preserved. The $\overrightarrow{0}$ remainder of the collateral branches of the aorta were of occluded $1 \mathrm{~cm}$. from their origin.

After irrigation of the specimen with normal saline at $37^{\circ}$ C. a suspension of micropaque was injected (diameter $0.5 \mu$ ) at manometrically controlled pressures $\bar{\partial}$ of $100 \mathrm{~mm}$. $\mathrm{Hg}$ before the fifth year and $120 \mathrm{~mm}$. $\mathrm{Hg}$ thereafter.

The arterial vasa were demonstrated by injecting through the brachio-cephalic trunk, the coronary sinus being occluded in neonatal and infant specimens to 3 prevent retrograde filling of the venous vasa in the adventitia of the pulmonary trunk with micropaque, which had tracked through the foramen ovale from the left side of the heart.

The venous vasa on the pulmonary trunk and $\stackrel{0}{x}$ arteries were injected through the coronary sinus and $\overline{0}$ bronchial veins respectively.

$X$-ray projection micrographs of full thickness pulmonary arterial wall, and about $1 \mathrm{~mm}$. thick sections cut by hand, were taken on Ilford contrasty plates with an exposure time of $5 \mathrm{~min}$. before the fifth year 3 and $7 \mathrm{~min}$. thereafter. The microscope was operated at $\rightarrow$ $10 \mathrm{kV}$ and $50 \mathrm{~mA}$ with an aluminium target providing the $\mathrm{X}$-radiation from birth until the fifth year, and $N$ at $15 \mathrm{kV}$ and $30 \mathrm{~mA}$ with a copper target from the fifth to fifteenth years.

When comparing the vascular densities of speci- N mens, the arterioles and capillaries were estimated 0 against a radio-opaque grid with 200 grid squares per inch, which was superimposed on the specimen when the micrograph was taken. The arterioles and capillaries in every square were counted as separate? vessels. Since the technical advantages of the $x$-ray $\frac{7}{0}$ microscope allow vessels in whole thickness mounts $\frac{\vec{D}}{\mathbb{D}}$ to remain in focus within limited magnifications, $\stackrel{\mathbb{D}}{\stackrel{D}{\perp}}$ vessel 'counts' were performed at primary magnifica- $\stackrel{\mathbb{Q}}{\mathbb{Q}}$ tions of $\times 20$. For ease of counting, such an $x$-ray micrograph may be projected on to a viewing screen and the number of vessels in each square of the grid $\delta$ 
may be recorded. The area of a full thickness mount of pulmonary wall covered by the grid at each mount was $0.5 \mathrm{~cm} .^{2}$, and a total of three such fields were examined from each of the pulmonary trunk, extrahilar and intrahilar pulmonary arteries. The site of these fields was kept constant in different specimens.

The density measurement per square of the grid in each part of the wall, therefore, came from a total of $1.5 \mathrm{~cm}^{2}$ of pulmonary wall and was obtained from the total number of vessels divided by the number of squares.

The intrinsic vascular arrangements were studied histologically in five neonates and in 10 specimens from children between the first and fifteenth years of life. Pickworth's (1934) method (sodium nitroprusside benzidene stain) and routine sections stained with Masson and Mallory's trichrome techniques were used to provide controls for the micrographs.

\section{RESULTS}

\section{RADIOLOGICAL OBSERVATIONS}

Pulmonary trunk In the neonatal pulmonary trunk it was found that an irregular plexus of arterioles, $40-60 \mu$ in diameter, originating from branches of the coronary arteries similarly to those of adults (Clarke, 1964b), existed in the adventitia of the pulmonary wall, being densest around the base and terminating on the pulmonary bifurcation in a sparse network (Fig. 1). In the infant pulmonary trunks the irregular plexus was less dense at the end of the first year, being replaced by longitudinal sinuous arterioles, $60 \mu$ in diameter, in the second year. In the fifth year there was an increase in the density of the longitudinal arteriolar pattern, with evidence of coiling of the arterial vasa at the base of the pulmonary trunk (Fig. 2).

From the adventitial arteriolar plexus arterial vasa penetrated the superficial layers of the outer third of the media in the fourth year to form a second network of vessels, $10-20 \mu$ in diameter (Fig. 3).

Examination of the venous vasa showed that an irregular plexus, densest in neonatal specimens, was formed in the adventitia until the fourth year, when small tributaries drained the outer third of the media to form longitudinally arranged adventitial veins, $50-70 \mu$ in diameter, which were tributaries of the ventricular cardiac veins (Fig. 4).

Pulmonary arteries In the neonatal pulmonary arteries it was found that an irregular network of arterioles, $40-50 \mu$ in diameter, originating from terminal branches of the bronchial arteries, was formed in the adventitia of the extrahilar parts of the pulmonary arteries, no injection medium entering the wall of the intrahilar parts (Fig. 5). In the infant pulmonary arteries, longitudinally arranged sinuous arterioles, $60-80 \mu$ in diameter, replaced the irregular adventitial network on the extrahilar part of the pulmonary arteries and extended onto the proximal part of the intrahilar pulmonary wall as straight arterioles, $30-50 \mu$ in diameter, in the second year (Fig. 6).

It was clear from the micrographs that by the fifth year the longitudinal arteriolar pattern on the extrahilar part of the pulmonary arterial wall had increased in density, and that the intrahilar part of the pulmonary artery had a longitudinally arranged plexus of arterioles in the adventitia, which terminated in branches $2 \mathrm{~mm}$. in diameter (Fig. 7).

From the adventitial arteriolar plexus arterial vasa penetrated the superficial layers of the media on the proximal parts of the extrahilar portion of the pulmonary artery in the fourth year, being confined to the adventitia elsewhere (Figs 3 and 8).

The micrographs showed that the venous vasa formed an irregular plexus, which was confined to the adventitia until the fourth year, when it drained the outer third of the media on the proximal parts of the extrahilar pulmonary arteries to form a network of veins, $80-100 \mu$ in diameter. Venous vasa appeared on the intrahilar parts of the pulmonary wall in the second year and remained adventitial (Fig. 9).

Pulmonary trunks and arteries from children between 5 and 15 years of age No alteration could be demonstrated in the pattern described for the pulmonary vasa until the tenth year.

Between the tenth and twelfth years the longitudinal adventitial arterioles in the adventitia of the pulmonary trunk showed increased sinuosity and coiling at the margins and base of the vessel, and mosaic patterns appeared on its anterior and posterior surfaces (Fig. 10). Arterial vasa began to penetrate the remainder of the outer third of the media in the tenth year, vascularization of this layer being complete by the fifteenth year.

Examination of the micrographs showed that there was an increase in the longitudinal venous channels in the adventitia of the pulmonary trunk in the twelfth year, tributaries draining into the adventitial veins from the middle third by the fifteenth year (Fig. 11).

The longitudinal adventitial arterioles on the extrahilar part of the pulmonary arteries showed coiling, while the arterioles in the adventitia of the intrahilar arteries became more sinuous (Fig. 12). 


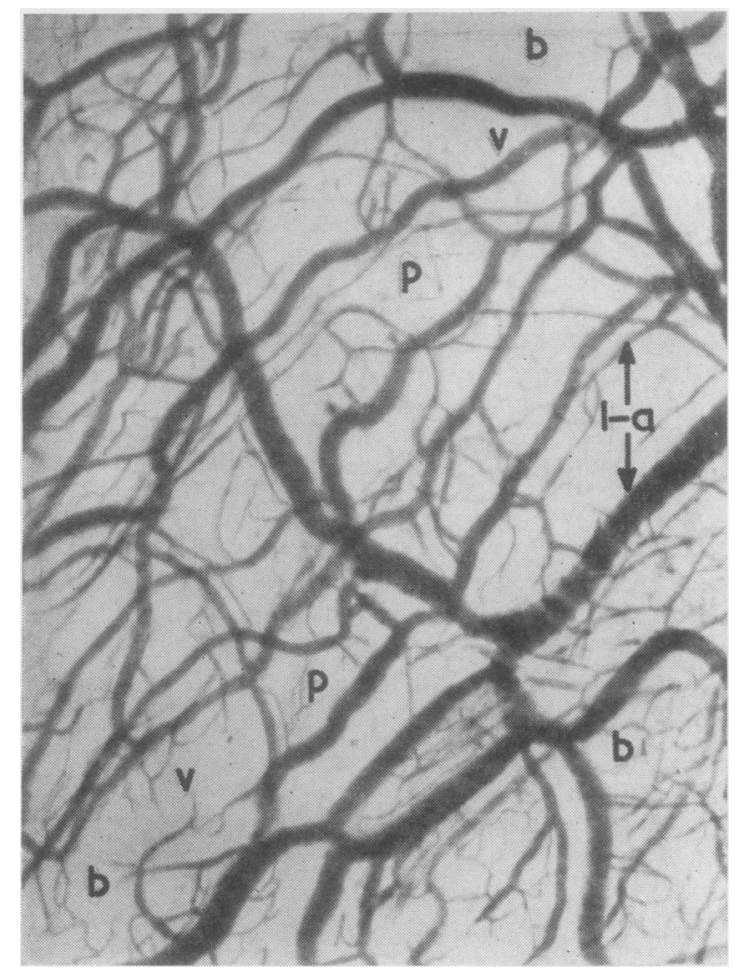

FIG. 1

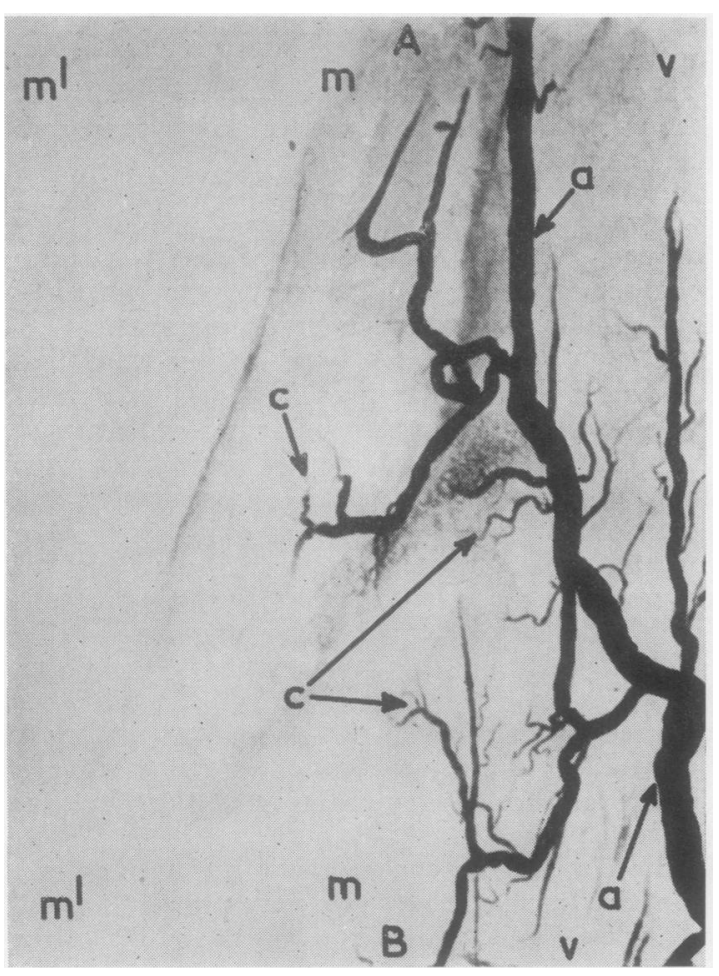

FIG. 3

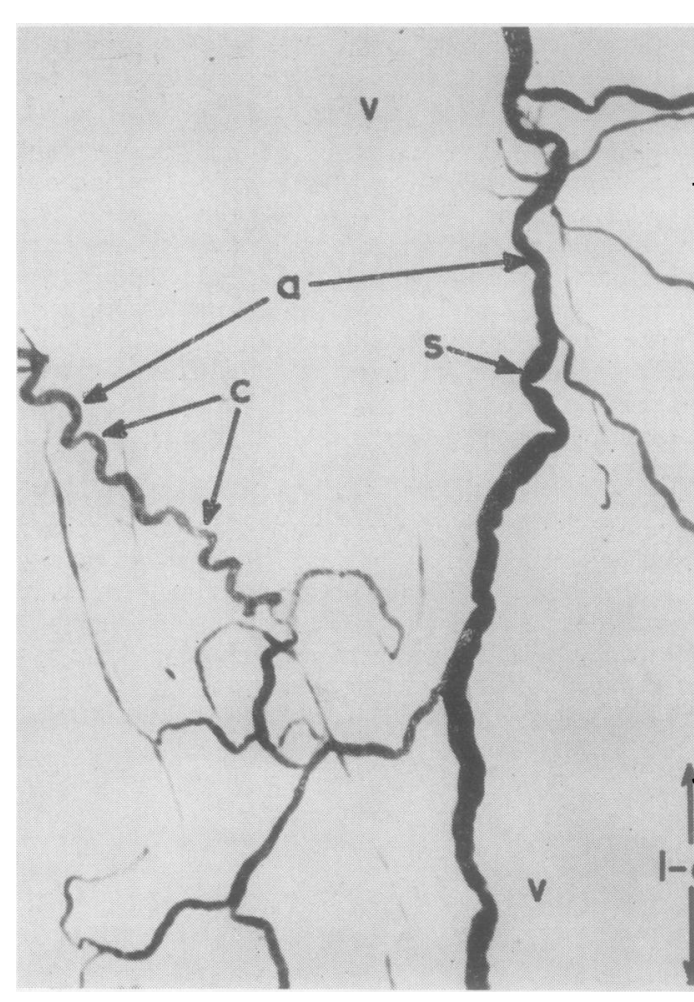

FIG. 2

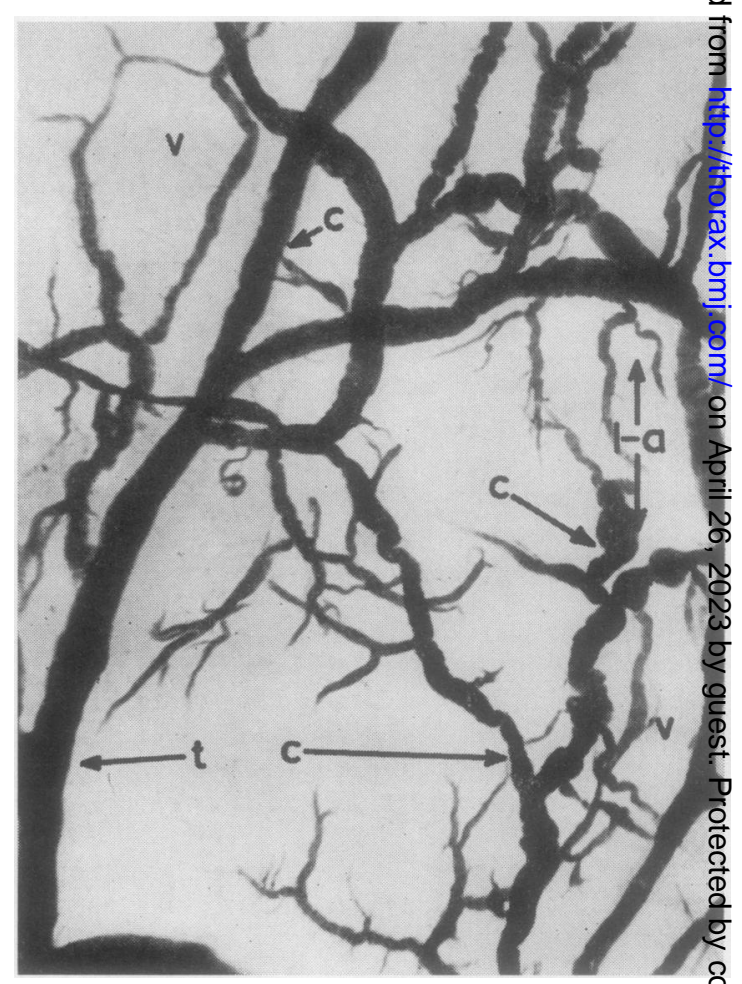

FIG. 4 


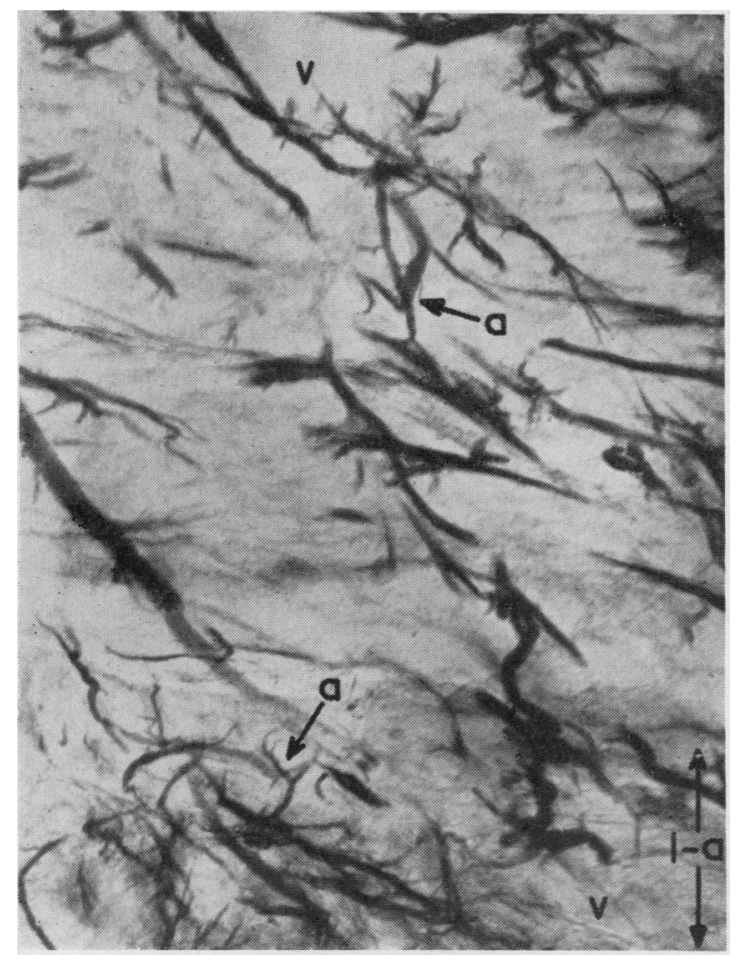

FIG. 5

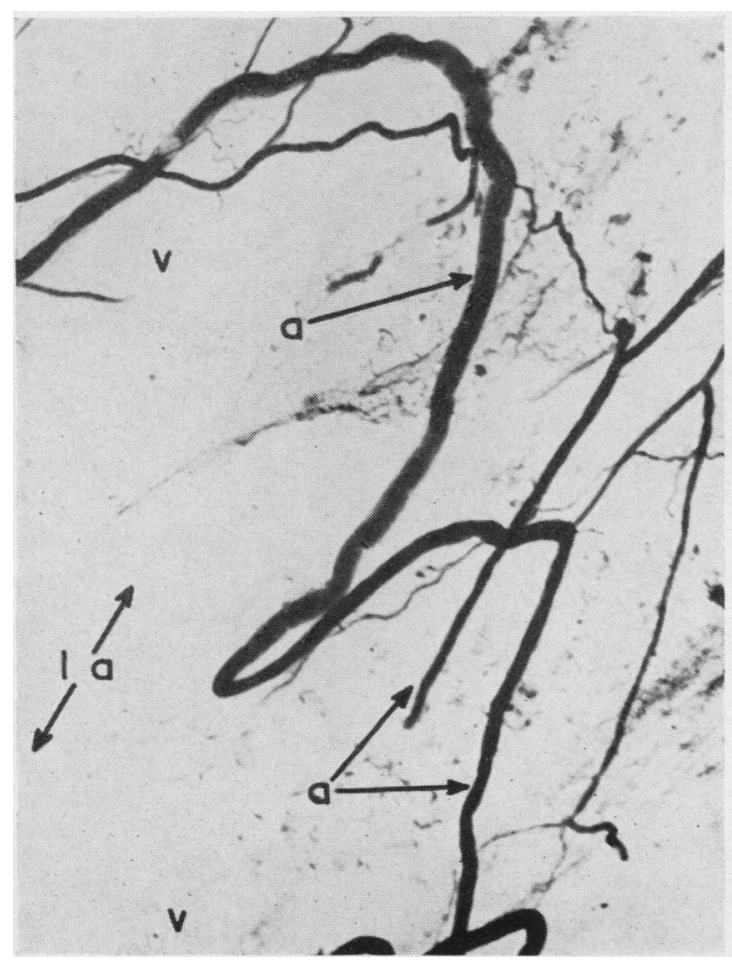

FIG. 7

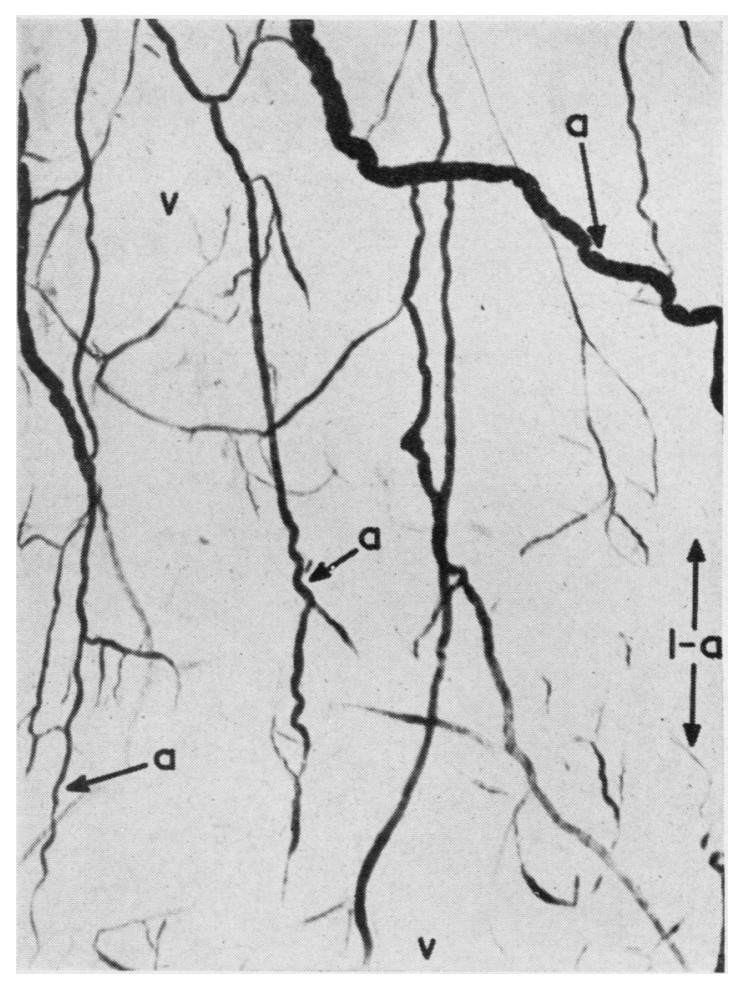

FIG. 6

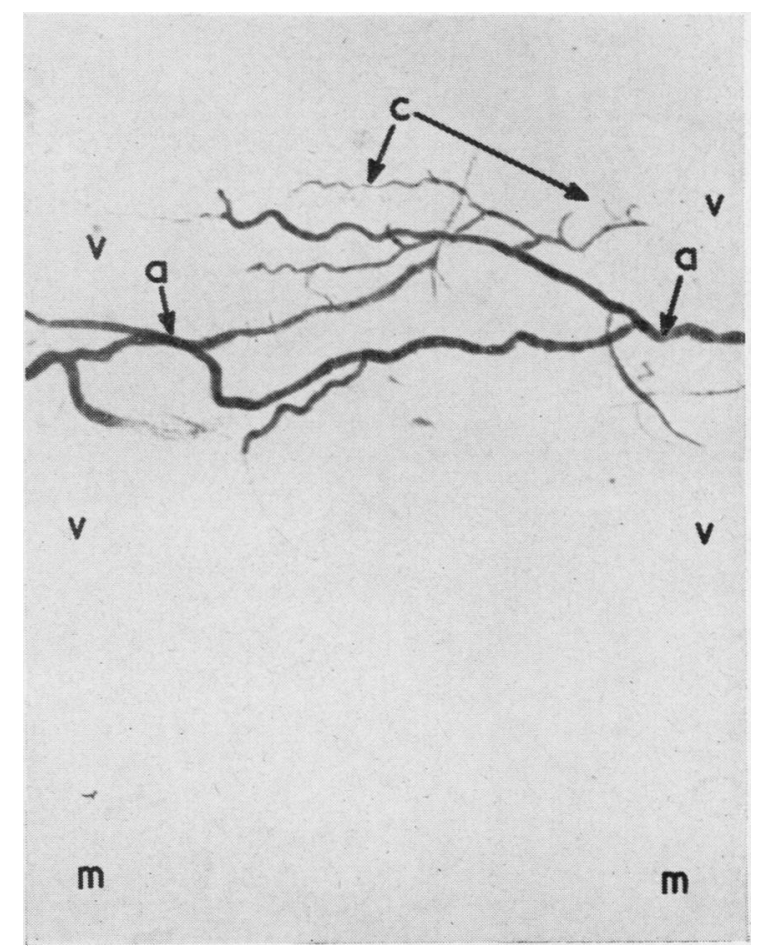

FIG. 8 


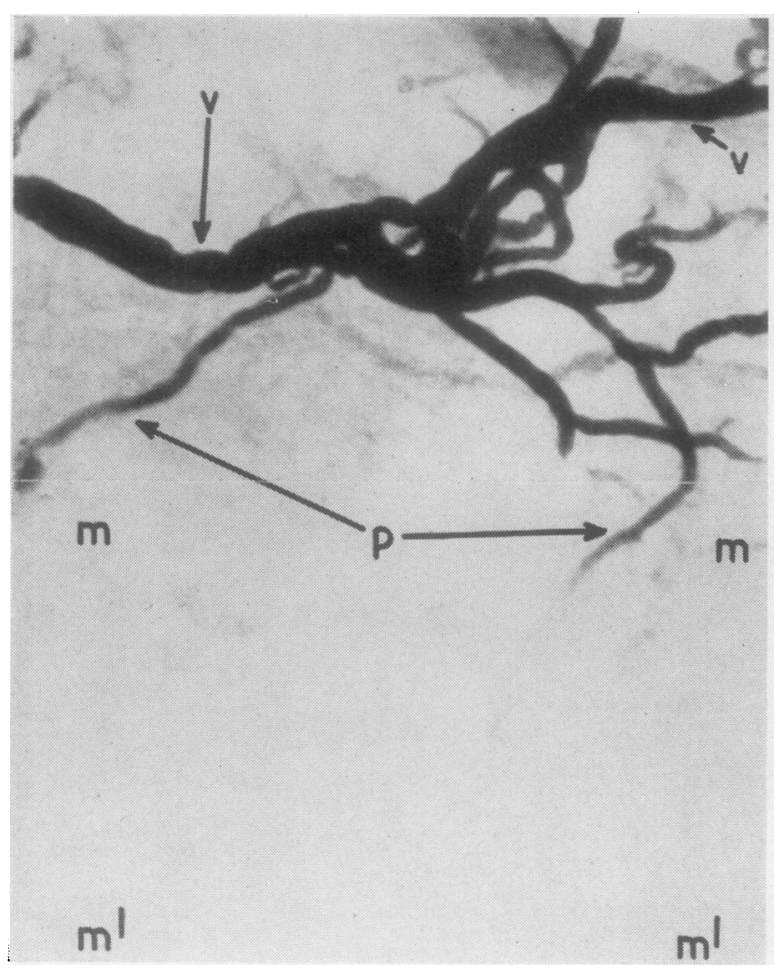

FIG. 9

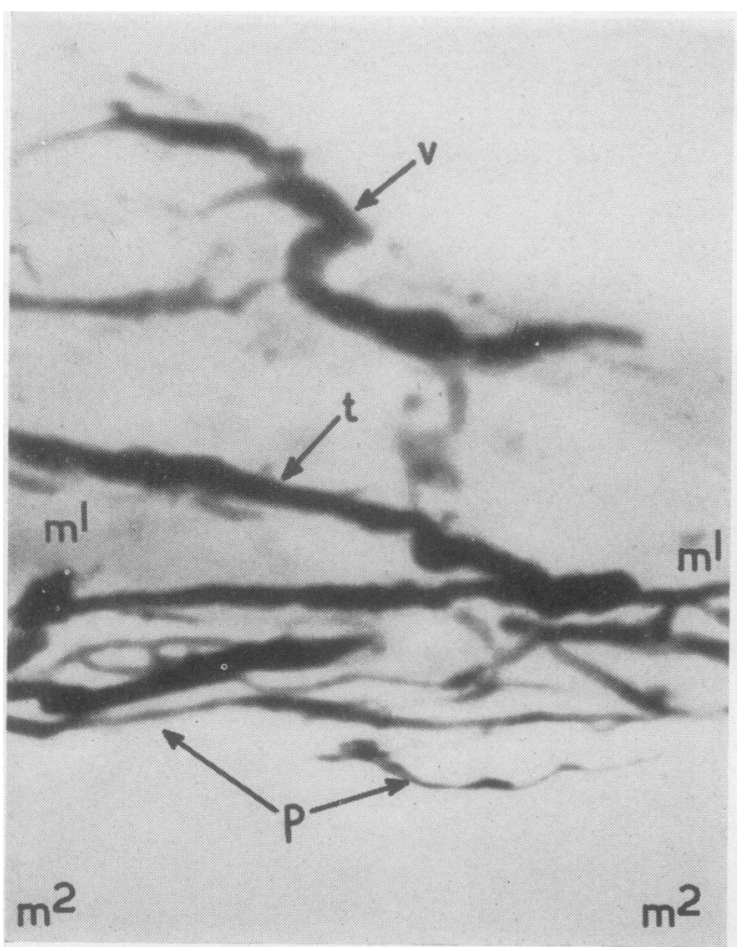

FIG. 11

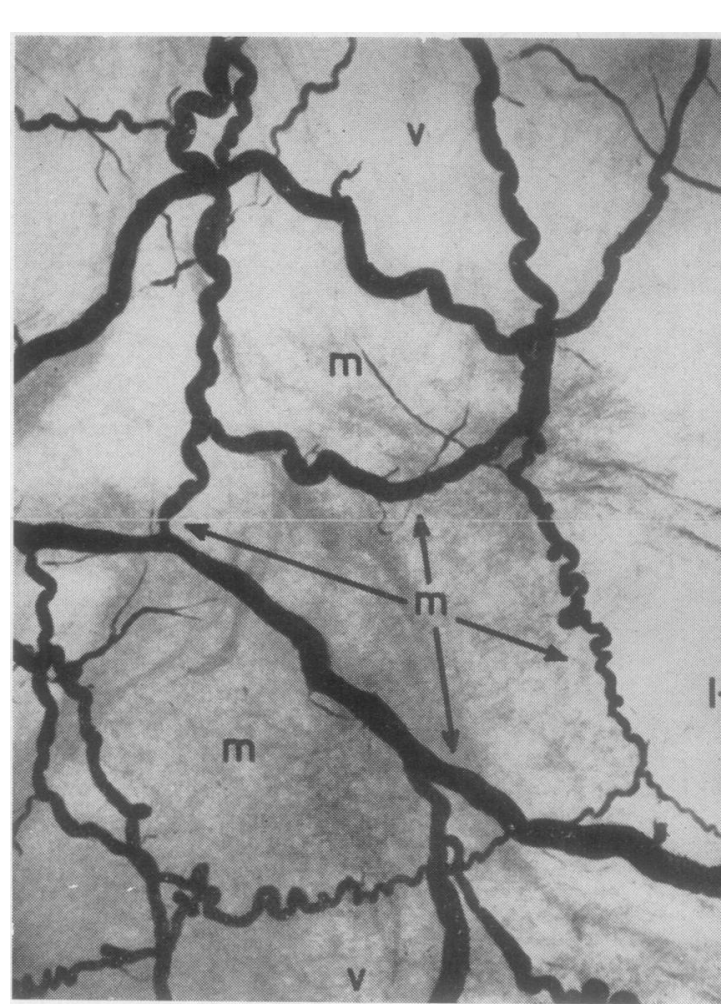

FIG. 10

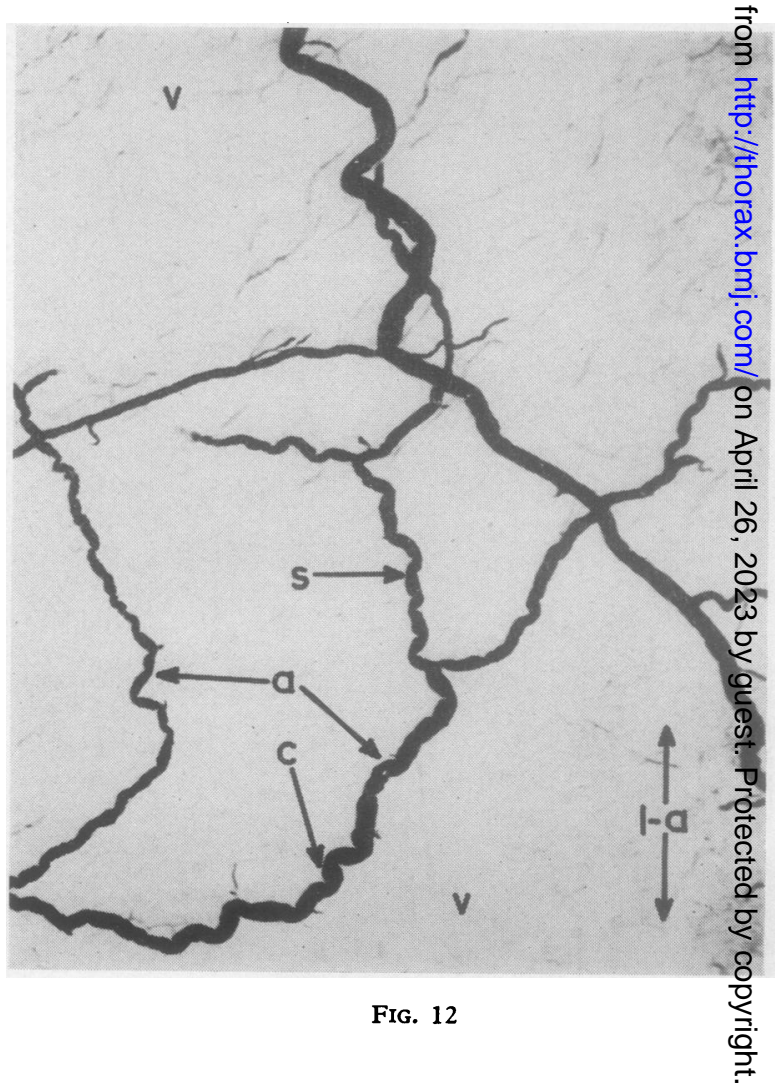




\section{EXPLANATION OF PLATES}

All micrographs are of full thickness pulmonary trunk and artery wall except Figs 3, 8, 9, 11, and 13.

FIG. 1. Micrograph of the base of the pulmonary trunk, aged 3 weeks. Note the irregular arteriolar plexus $(p)$ around the base of the pulmonary trunk (b). Adventitia (v), $\times 50$.

FIG. 2. Micrograph of the pulmonary trunk, aged 5 years. Note the sinuosity $(s)$ and coiling $(c)$ of the arterioles $(a)$ in the adventitia $(v), \times 40$.

FIG. 3. Micrograph of $1 \mathrm{~mm}$. thick longitudinal section of the junction of the pulmonary bifurcation $(A)$ and the proximal part of the right pulmonary artery $(B)$, aged 4 years. Note the arterioles $(a)$ in the adventitia $(v)$ penetrating and supplying capillaries (c) to the outer third of the media $(m)$. No injection medium has entered the middle third of the media $(m 1), \times 100$.

FIG. 4. Micrograph of the pulmonary trunk, aged 5 years. Note the longitudinal venous channels $(c)$ in the adventitia (v) draining into tributaries $(t)$ of the ventricular cardiac veins, $\times 60$.

FIG. 5. Micrograph of the right pulmonary artery, aged 3 weeks. Note the irregular plexus of arterioles $(a)$ in the adventitia $(v), \times 40$.

FIG. 6. Micrograph of the right pulmonary artery, aged 2 years. Note the sinuous arterioles $(a)$ in the adventitia $(v)$, $\times 30$.

Arterial vasa penetrated the outer third of the media in the distal parts of the extrahilar pulmonary arteries in the tenth year, vascularization of this layer being complete by the fifteenth year. The arterial vasa were confined to the adventitia of the intrahilar pulmonary arteries, an increase in the density of the vascular pattern being the only change observed.

The venous vasa retained the irregular pattern seen in the infant pulmonary arteries, tributaries draining into the adventitial plexus from the junction of the outer and middle thirds of the extrahilar arteries but being confined to the deep adventitial layers in the intrahilar arteries (Fig. 13).

No injection medium entered the intima of any specimen examined.

Histological observations In general the vasa vasorum conformed to previous descriptions of neonatal, infant, and children's specimens, but the pattern of distribution could not be appreciated (Fig. 14).

Vascular densities in pulmonary arterial wall When the arteriole and capillary distribution was
FIG. 7. Micrograph of the upper lobar branch of the left pulmonary artery, aged 5 years. Note the longitudinally placed arterioles $(a)$ in the adventitia $(v), \times 45$.

FIG. 8. Micrograph of $1 \mathrm{~mm}$. thick longitudinal section of the left upper lobar pulmonary artery, aged 4 years. Note the arterioles $(a)$ and capillaries $(c)$ in the adventitia $(v)$. No injection medium has entered the media $(m), \times 100$.

FIG. 9. Micrograph of $1 \mathrm{~mm}$. thick longitudinal section of the proximal part of the right pulmonary artery, aged 4 years. Note the origin of the venous plexus $(p)$ in the outer third of the media $(m)$ draining into adventitial veins $(v)$. No injection medium has entered the middle third of the media $(\mathrm{m} 1), \times 100$.

FIG. 10. Micrograph of the posterior aspect of the pulmonary trunk, aged 10 years. Note the arteriolar mosaics $(m)$ in the adventitia $(v), \times 30$.

FIG. 11. Micrograph of $1 \mathrm{~mm}$. thick transverse section of the pulmonary trunk, aged 15 years. Note the origin of the venous plexus $(p)$ in the middle third of the media $(m 1)$ draining into tributaries $(t)$ of the adventitial veins $(v)$. No injection medium has entered the inner third of the media (m2), $\times 110$.

FIG. 12. Micrograph of the left pulmonary artery, aged 10 years. Note the increased sinuosity $(s)$ and beginning of coiling $(c)$ of the arterioles $(a)$ in the adventitia $(v), \times 35$.

examined on the grid, it was found that the density of the arterioles and capillaries was greater during the neonatal period than at the end of the first year of life in the pulmonary trunk. The results of arteriole and capillary 'counts' recorded in different parts of the pulmonary arterial wall between birth and 15 years of age are shown in Figure 15.

\section{DISCUSSION}

The technical difficulties encountered in demonstrating the patterns of the vasa vasorum constitute a limiting factor when studying the vessels in the wall of the pulmonary arterial tree.

In contrast to injection methods, techniques which require the staining of red cells depend on the uniform filling of the capillaries with red cells at the time of death, and the failure to demonstrate vasa vasorum by Pickworth's (1934) method may be due to a lack of blood within the mural vessels. Demonstration of vasa by routine staining techniques or by examining alkaline phosphatase in endothelial cells does not allow the pattern of the vasa vasorum to be appreciated, in contrast to the routine $x$-ray micrograph. 


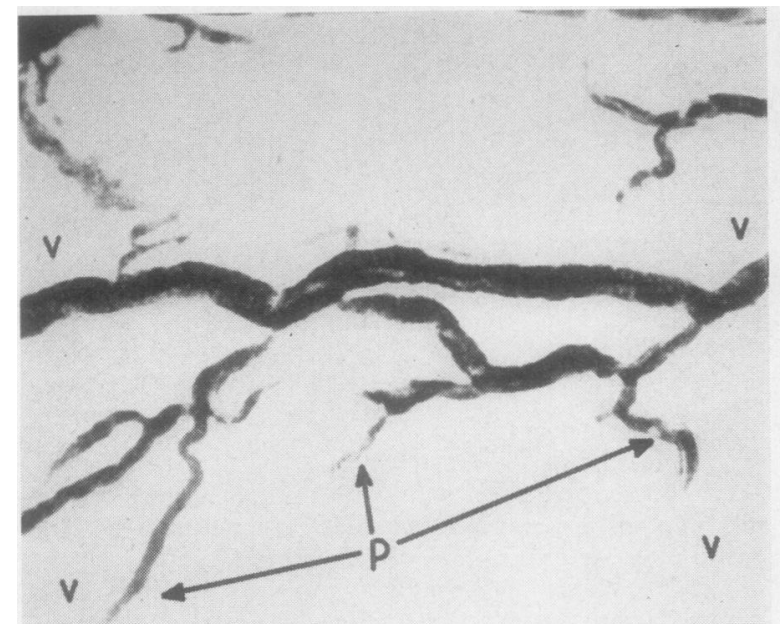

n

nn

FIG. 13

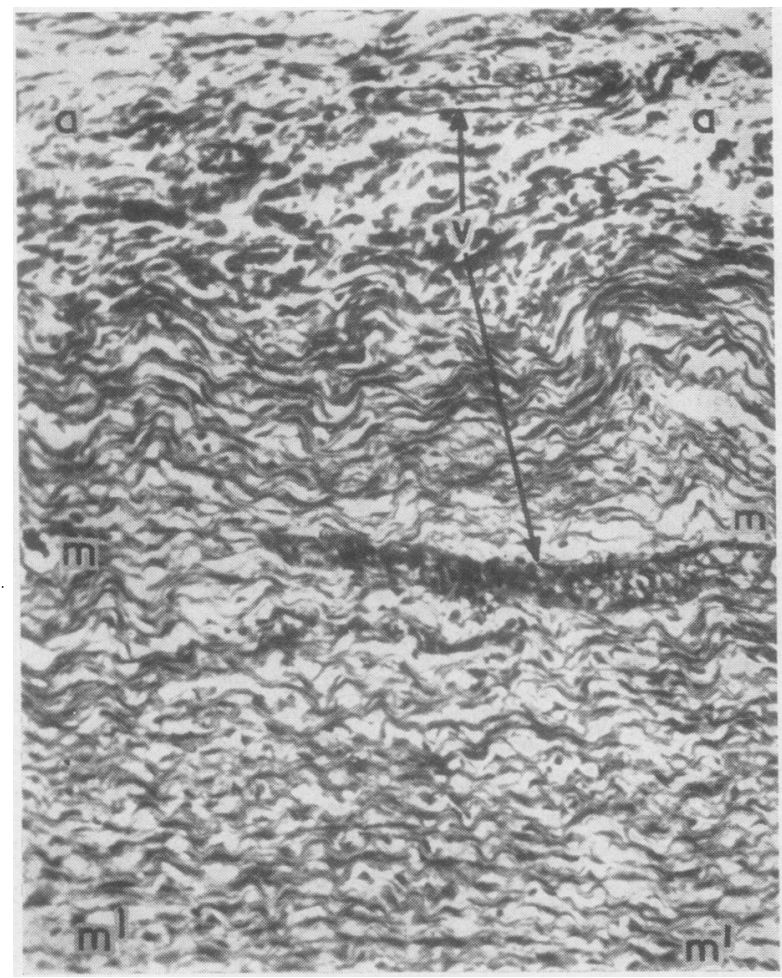

FIG. 14
FIG. 13. Micrograph of $1 \mathrm{~mm}$. thick longitudinal section of the lingular branch of the pulmonary artery, aged 0 14 years. Note the origin of the venous plexus (p) in the $\bar{\sigma}$ deep layers of the adventitia (v). No injection medium has $\overline{\bar{\omega}}$ entered the media $(m), \times 110$. of $8 \mu$ thick longitudinal section of the left pulmonary artery, aged 15 years. Note the vasa vasorum $(v)$ in the adventitia (a) and outer third of the $\vec{\circ}$ media (m). No vasa can be seen in the middle third of the media (m1). Mallory, $\times 100$.

FIG. 15. Histogram showing the arteriole and capillary $\overrightarrow{\dot{x}}$ 'counts' in the wall of the pulmonary arterial tree between birth and 15 years of age.

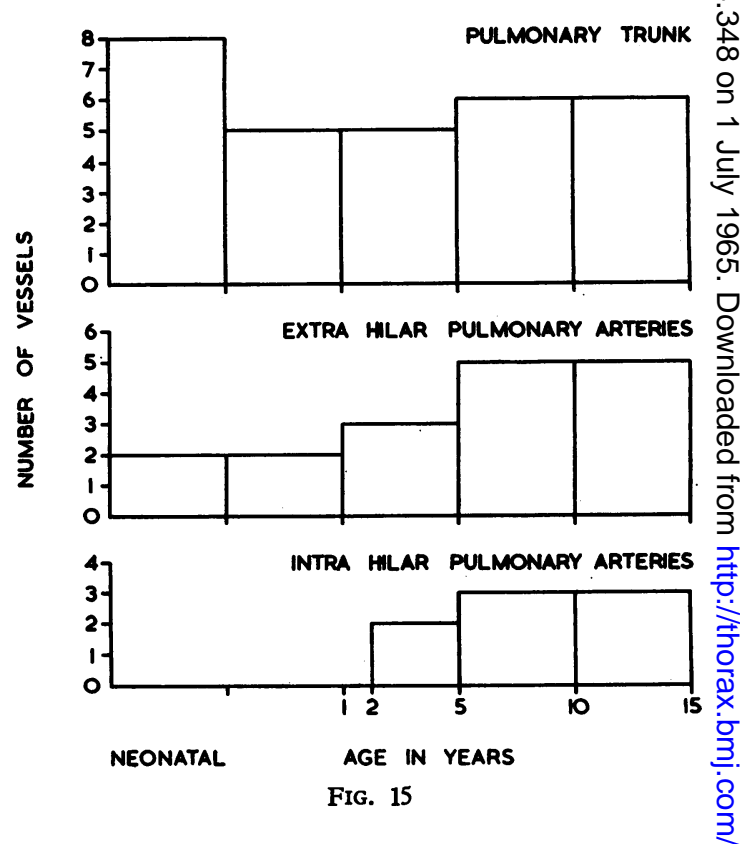

Staining methods are suited to the investigation of adaptive changes in the circulation, demon- $\rightarrow$ strating the actual state of the circulation at that time. Injection methods, on the other hand, tend $\bar{N}$ to present the vascular pattern at its maximum capacity and are particularly suited for anatomical $N$ investigations. For this reason, along with the ${ }_{\omega}^{N}$ technical advantages of $x$-ray microscopy, which 0 allows an examination of small arterioles and capillaries in unfixed, full thickness specimens, the $\frac{\text { }}{\varnothing}$ vasa vasorum of the aorta were investigated by $\stackrel{\infty}{\rightarrow}$ this method.

In this study the criterion for accepting an area $\bar{O}$ of the young pulmonary arterial wall as devoid of $\stackrel{\odot}{\circ}$ vasa vasorum was the repeated failure to introduce $\vec{D}$ the injection medium into the arterial wall under $\frac{\varrho}{\sigma}$ conditions which filled capillary beds elsewhere in the same specimen, and subsequently provedo 
satisfactory in these areas in older specimens. When assessing the depth the vasa vasorum penetrated, specimens showing 'stub end' arterioles were discarded. Only when a reasonable extent of the capillary bed had been revealed was any interpretation made, and, although 'stub ends' of capillaries were observed, it was felt that this was inevitable with any injection method sooner or later, no matter how 'complete' it was.

The present study has shown that there is an extensive vascular supply to the pulmonary arterial wall, the arrangement and distribution of which alter considerably in early postnatal life.

The first year was characterized by an irregular network of arterial vasa on the pulmonary trunk and extrahilar part of the pulmonary arteries, the former being reduced in vascular density towards the end of the first year. The pattern was similar to the arrangement of vessels on the pulmonary trunk in the later months of intra-uterine life but differed from the pattern in the foetal pulmonary arteries, which were almost avascular (Clarke, 1965c).

The rapid vascularization of the distal part of the extrahilar pulmonary arteries in the neonatal period contrasted sharply with the foetal picture, in which a few vessels spread onto the pulmonary arteries from the pulmonary bifurcation. This was interpreted as a nutritional necessity for the pulmonary wall meeting the adapted circulation. The reduction in vasculature of the pulmonary trunk towards the end of the first year was regarded as the first stage in the alteration of the vasal architecture to the adult form.

The second year was characterized by the appearance of longitudinally arranged sinuous arterioles on the pulmonary trunk and extrahilar pulmonary arteries, with an extension onto the intrahilar pulmonary arteries. These changes occurred constantly in specimens between the ages of 18 and 24 months, and were interpreted as the templates for the formation of adult patterns.

Between the second and fifth years the appearance of the arterial vasa on the pulmonary arteries resembled the adult picture more closely, and the intrahilar pulmonary arterial wall received an ingrowth of vessels which was confined to the adventitia. The longitudinal arterioles in the adventitia of the pulmonary trunk were similar to those of the adult pattern and showed coiling in the vessels at the base, but there was no evidence of the formation of the mosaic patterns seen on the anterior and posterior surfaces in later life.

Penetration of the superficial layers of the outer third of the media occurred in the fourth year in the pulmonary trunk and the proximal parts of the extrahilar pulmonary arteries. This was similar to the development of the intramural pattern seen in the ascending aorta (Clarke, 1965d).

The tenth to twelfth years of life were characterized by increased coiling of the adventitial arterioles at the base and margins of the pulmonary trunk and on the extrahilar part of the pulmonary arteries. In addition, the adventitial arterioles on the intrahilar arteries showed increased sinuosity. The mosaic patterns on the anterior and posterior surfaces were formed at this time and appeared to emerge from a cross anastomotic network between the existing longitudinal arterioles in the adventitia.

Between the thirteenth and fifteenth years the arterial patterns which were forming in the adventitia from the tenth year were completed, and the adult picture was simulated.

Penetration of the outer third of the media in the distal part of the extrahilar arteries began in the tenth year and was complete by the fifteenth year, but the arterial vasa remained in the adventitia of the intrahilar arteries.

The latent period between the fifth and tenth years in the development of the vascular patterns is similar to that of the aorta (Clarke, 1965d). In the tenth year the mural vessels alter their characteristics, as do the aortic vasa, and this appears to provide for the growth of the pulmonary trunk and ascending aorta at this time.

On account of the relatively small size of the sample studied (specimens of this age group unaffected by cardiovascular disorders are difficult to obtain) these observations on the progressive vascularization of the pulmonary arterial wall could be interpreted as a failure of injection technique. On the other hand, the number and thickness of the elastic membranes in the aorta and pulmonary trunk gradually increase after birth (Bloom and Fawcett, 1962), and the ingrowth of vessels may be a nutritional necessity accompanying the increased girth of these vessels.

The venous vasa of the pulmonary trunk and arteries were found to vary little after the second year. Before this time the intrahilar arteries were avascular, but with the ingrowth of arterioles to the adventitia, an accompanying plexus of veins appeared. The veins in the walls of the pulmonary trunk and arteries were tributaries of the ventricular cardiac veins and bronchial veins respectively. The venous vasa showed little change between the tenth and fifteenth years apart from an increase in the longitudinal venous channels in the adventitia of the pulmonary trunk, and cross anastomoses with the ascending aortic venous vasa were formed. 
By the fifteenth year vessels $10-20 \mu$ in diameter could be demonstrated in the middle third of the media of the pulmonary trunk when the venous vasa were injected. In contrast, injection of the arterial vasa only demonstrated a capillary plexus at the junction of the superficial and middle thirds of the media. The deeper penetration of the injection mass when the venous route was used was a constant finding in all parts of the pulmonary arterial tree. It was concluded, therefore, that the capillary-venule bed of the pulmonary trunk lay in the middle third of the media. As shown by the $x$-ray microscope, the capillary-venule bed of the extrahilar pulmonary arteries lay in the outer third of the media and in the deep layers of the adventitia of the intrahilar arteries.

As indicated by the $x$-ray microscope, the neonatal distribution of arterioles and capillaries was denser in the pulmonary trunk than at the end of the first year of life. In contrast, the vessel 'count' in the extrahilar part of the pulmonary arteries was the same at the end of the first year. Thereafter the arteriole and capillary density increased in the pulmonary trunk from the fifth year-from the end of the first year on the extrahilar arteries and from the end of the second year on the intrahilar arteries. By the fifteenth year the pulmonary trunk showed the greatest vascularity of any part of the pulmonary arterial tree. After the fifteenth year there was no change in the arteriole and capillary 'counts'. Since the sample studied was small, no formal statistics could be performed, and these observations can only be regarded as a guide to the range of variation which appears to occur.

It appears that the morphological pattern and distribution of the vasa vasorum approximate to the adult picture by the fifteenth year, and it was concluded that maturity of vasal architecture had been achieved by the limit of the age group studied.

\section{SUMMARY}

The arterial and venous vasa of the pulmonary trunk and arteries were examined from patients between birth and 15 years of age.

The arterial vasa of the pulmonary trunk originated from branches of the coronary arteries and the arterial vasa of the pulmonary arteries from terminal branches of bronchial arteries.

The venous vasa of the pulmonary trunk drained into ventricular cardiac veins, the venous vasa of the pulmonary arteries into tributaries o $\overrightarrow{\vec{B}}$ the bronchial veins.

The arterial vasa penetrated the outer third of the media in the pulmonary trunk from the fourthe year and the outer third of the media in the dista $\mathbb{B}$ part of the extrahilar pulmonary arteries from the tenth year. Arterial vasa were confined to the adventitia of the intrahilar arteries.

Venous vasa originated in the middle third of $\overrightarrow{\dot{\omega}}$ the media in the pulmonary trunk, in the outero third of the media in the extrahilar pulmonary㞔 arteries, and in the adventitia of the intrahilarin arteries.

The capillary-venule bed lay in the middle thirct of the media in the pulmonary trunk, in the outeros third of the media in the extrahilar arteries, ando in the adventitia of the intrahilar arteries.

Various patterns were found in the arterial and venous vasa as the pulmonary arterial tree developed, adult appearances being demonstrable by the fifteenth year.

No injection medium entered the intima.

Arteriole and capillary counts of the variouso parts of the pulmonary tree were recorded for thes different age groups.

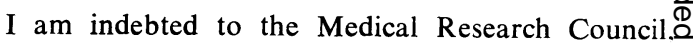
who provided the $x$-ray microscope for the Anatomy $\overrightarrow{\vec{O}}$ Department, Glasgow University.

\section{REFERENCES}

Bloom, W., and Fawcett, D. W. (1962). A Textbook of Histology 8 th ed. Saunders, London and Philadelphia.

Clarke, J. A. (1964). An x-ray microscopic study of the vasa vasorume of the normal human pulmonary arteries. Thorax, 19, 561.

(1965a). An x-ray microscopic study of the vasa vasorum of thehuman ductus arteriosus. J. Anat. (Lond.). In press.

(1965b). An x-ray microscopic study of the vasa vasorum of the human pulmonary trunk. Acta anat. (Basel). In press. (1965c). An X-ray microscopic study of the vasa vasorum of theo human foetal aorta and pulmonary trunk. Ibid. In press. (1965d). An X-ray microscopic study of the postnatal develop ment of the vasa vasorum in the aorta. $J$. Anat (Lond In press $D$

O'Neill, J. F. (1947). The effects on venous endothelium of alterations in blood flow through the vessels in vein walls, and the possiblerelation to thrombosis. Ann. Surg., 126, 270. and the possible

Pickworth, F. A. (1934). A new method of study of the brain capilo laries and its application to the regional localisation of mentah disorder. J. Anat. (Lond.), 69, 62.

Scharrer, E. (1950). A technique for the demonstration of the blood vessels in the developing central nervous system. Anat. Rec. $\omega$ $107,319$.

Schlichter, J. G. (1948). Vascularization of the aorta in differento

williams beds by intravascular precipitation of 100, 115 . Anat. Rec.

Winternitz

The Biology of Arteriosclerosis. Charles C. Thomas, Springfield The Biology of Arteriosclerosis. Charles C. Thomas, Springfield,

Woerner, C. A. (1959). In The Arterial Wall, ed. A. I. Lansing. Chap pp. 1-14. Williams and Wilkins, Baltimore. 